\title{
CODE TRACKING OF DSSS SIGNAL OVER AWGN CHANNEL
}

\author{
MOHANAD ABDULHAMID ${ }^{1}$, HASSAN OMAR ${ }^{2}$ \\ ${ }^{1}$ AL-Hikma University, Iraq, ${ }^{2}$ University of Nairobi, Kenya \\ E-mail: moh1hamid@yahoo.com, researcher12018@yahoo.com
}

\begin{abstract}
Direct sequence spread spectrum (DSSS) communication systems offer significant performance advantages in view of their low probability of intercept, improved performance in multipath fading environments and their ability to avoid interference by spreading the signal over a wide bandwidth hence distributing the power. For the transmitted sequence to be correctly received and demodulated, the spreading sequence used at the receiver should be similar to that employed in the transmitter. This paper utilizes MATLAB Simulink to demonstrate a method of synchronizing the code clock at the receiver with the code clock at the transmitter. This fine alignment process is known as code tracking.
\end{abstract}

Keywords: DSSS system, code tracking, AWGN channel

\section{INTRODUCTION}

Spread spectrum communications refers to the technique of taking an information bearing signal of a particular bandwidth and deliberately spreading it out on the frequency domain such that the signal now occupies a much wider bandwidth $[1,2]$.

There are three main techniques utilized in spread spectrum communications. Direct sequence spread spectrum, frequency hopping spread spectrum, and time hopping spread spectrum. With each utilizing a code that is pseudorandom in nature to achieve the spreading $[3,4]$.

Each system of spreading the spectrum requires a method of recovering the transmitted signal. This requires the receiver to recover the spreading code timing from the received signal. The acquisition system brings the receiver and transmitter code sequences into coarse alignment while tracking the system [5].

In DSSS system, spreading is achieved by representing each bit of the original message sequence using several bits. This process is achieved by using a spreading code in this case a pseudo-noise (PN) sequence.

The PN sequence is XOR-ed with the message sequence. As a case in point, consider a case where the PN sequences are produced at a frequency that is 10 times higher than the message frequency. In this case, the message frequency will be spread by a factor of 10 after the XOR process. This step details the transmission process in a DSSS system [6,7].
At the receiver, the transmitted signal is again XOR-ed using a PN sequence that is similar to the one originally used at the transmitter. In this way the original message sequence can be recovered.

However, channel imperfections bring in the need for synchronization of the spreading sequence used at the transmitter, and the one employed at the receiver. This makes the code synchronization steps of code acquisition and code tracking important. In code acquisition, the PN sequence employed at the transmitter and the one employed at the receiver are brought to within one time period of the original spreading sequence. In code tracking, the PN sequence at the receiver that has already been brought to within one time period of that used at the transmitter by code acquisition is further refined and its accuracy is brought to within less than half of the time period of the code employed at the transmitter [6,7]. In practice code acquisition takes place before code tracking after which code tracking is initiated. In effecting this whole process a special algorithm that initiates the tracking phase as soon as acquisition is achieved is used.

The basis for code acquisition is correlating both the PN sequences used at the transmitter and the receiver and setting a threshold which once attained, acquisition is declared to have occurred. PN sequences are the most effective spreading sequences since as will be highlighted later they have maximum correlation when both PN sequences are in synchronization and minimum correlation which remains constant when the signals are out of synchronization [7].

The code acquisition process takes place over a finite amount of time. When longer PN sequences are used the acquisition time may be longer. In order to appreciate this fact, a brief description of what acquisition involves is necessary.

During acquisition, the PN sequence received from the receiver is compared with the one that is to be employed at the transmitter. If the correlation is below a set threshold, the PN sequence at the receiver is delayed by a single time period of the PN sequence. This process goes on recursively until a set threshold is attained. Once this threshold is attained, acquisition is said to have occurred [7].

The paper concentrates on demonstrating a means of code tracking on DSSS systems. The tracking deals with 
correction of timing offsets and frequency offsets of the clock running the code generators. Some researches which deals with tracking of DSSS can be found in literatures $[8,9,10,11]$.

The aim of this paper is to study spread spectrum communication systems with particular emphasis on direct sequence systems. This involves studying of code tracking in DSSS systems showing its necessity.

\section{DESIGN METHODOLOGY}

The tracking system is implemented using MATLAB Simulink. The complete model overview is shown in Figure 1, with each block is explained in the following subsections.

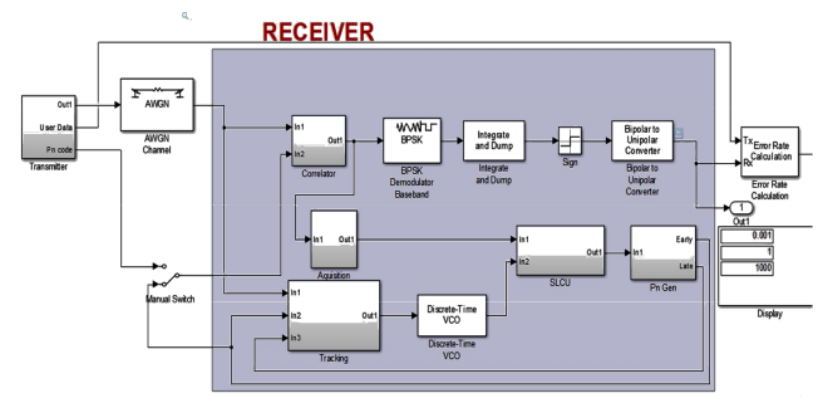

Figure 1. System block diagram

The following are the specifications of the system designed:

1 - Data rate: $1 \mathrm{~Kb} / \mathrm{s}$.

2 - PN code rate: $32 \mathrm{KHz}$.

3 - PN code length: 7 bits (maximal linear code).

4 - Modulation type: equivalent baseband binary phase shift keying (BPSK).

5 - Acquisition method: serial search (dwell time scheme).

6 - Tracking method: delay locked loop (DLL).

7 - Processing gain: $15.05 \mathrm{~dB}$.

\subsection{Transmitter}

The transmitter shown in Figure 2 consists of a data generator, a PN generator, a mixer and a baseband BPSK modulator. Since the modulation is performed in baseband, no radio frequency (RF) carrier is present therefore the detection of the signal in the receiver uses low pass filter.

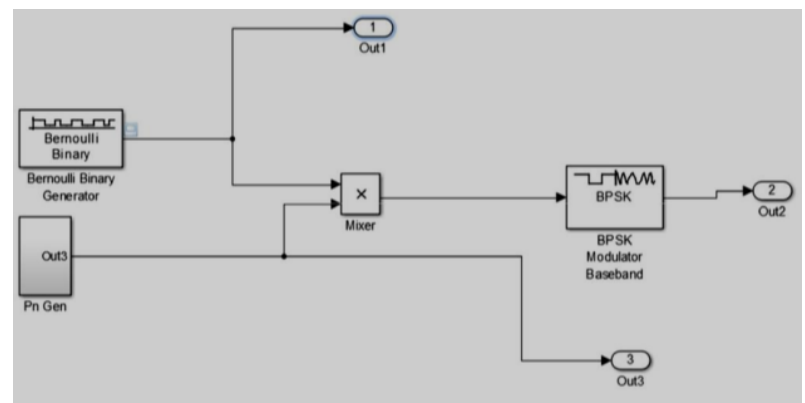

Figure 2. Transmitter block diagram

\subsubsection{Data Generator}

The data generator used is a Bernoulli data generator from the communication blockset with a $50 \%$ probability of a zero and $50 \%$ probability of a one.

\subsubsection{PN Generator}

The PN generator implemented had a minimal polynomial given by $\mathrm{fx} 1 \mathrm{xx}$. The $\mathrm{PN}$ is generated by using three stages D Flip-Flop from Simulink-Extras with a two feedback taped at $\mathrm{x}$ and $\mathrm{x}$, Ex-ORed to the input of the first stage D Flip-Flop, in order to get 7 bit length maximal (2 1) as shown in Figure 3.

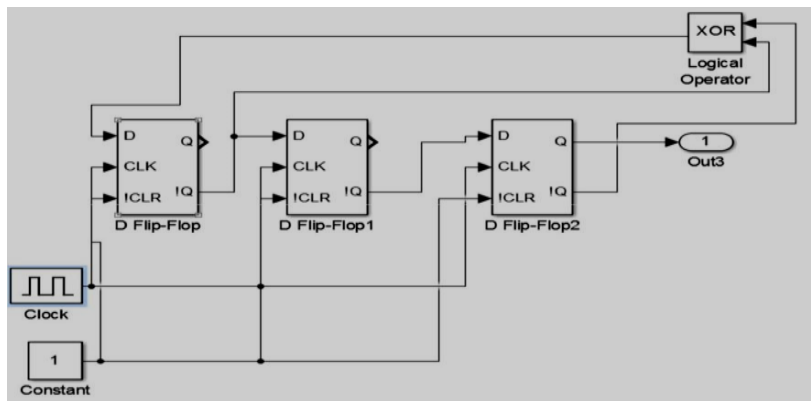

Figure 3. PN generator

\subsubsection{BPSK Modulator}

Here, the signal is modulated using binary phase shift keying from the mixer. This converts the signal to bipolar with a reverse phase of either 0 or 180 degrees.

\subsection{Channel}

The channel is modeled as an AWGN which is an additive white Gaussian noise channel. This models the channel as having corrupting noise being added to the signal in the process of transmission.

\subsection{Receiver}

The receiver consists of active correlator, synchronization unit, baseband BPSK demodulator, and local PN code generator with a variable clock, as shown in Figure 4.

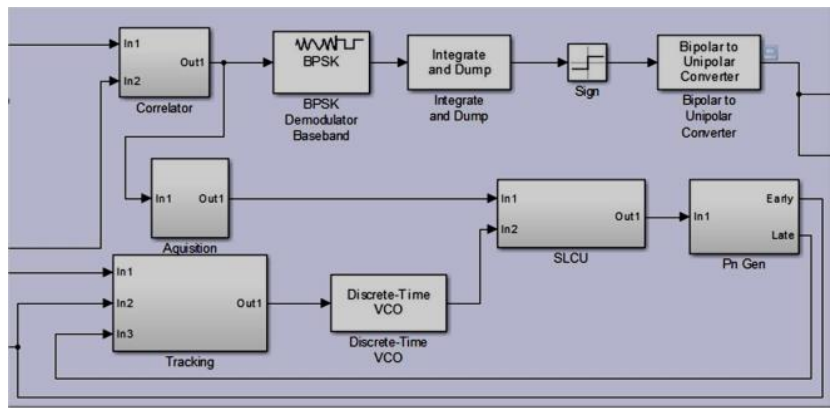

Figure 4. Receiver block diagram 


\subsubsection{Active Correlator}

It is made up of a mixer and a fourth order Butterworth filter to de-spread the signal. The received signal is only de-spread when the locally generated PN sequence and the received PN code have the same phase. The cutoff frequency of the filter is set to the data rate of $1 \mathrm{KHz}$. The implemented active correlator is as shown in Figure 5. The manual switch is used to measure the performance when using a digital filter and an integrator in the circuitry.

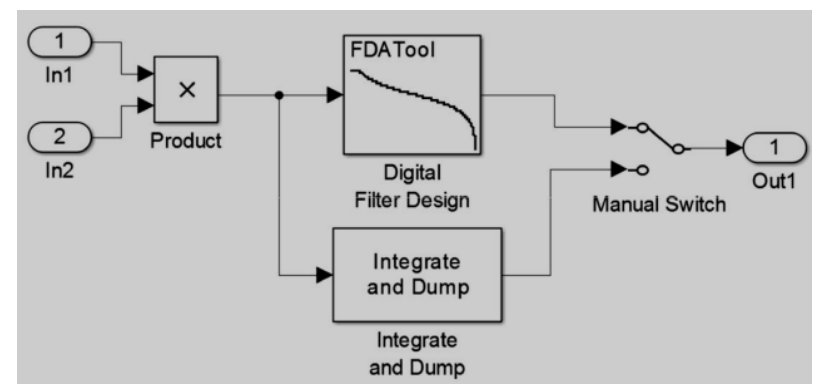

Figure 5. Active correlator

\subsubsection{Synchronization unit}

The synchronization unit consists of acquisition, tracking, voltage controlled oscillator (VCO), and search and lock control unit (SLCU).

The output of the active correlator is compared with a threshold level in acquisition, if the threshold is exceeded, no delay will be introduced to the local PN generator clock. This will mean that initial course synchronization has been achieved and tracking (fine synchronization) will be commenced. If the threshold is not reached, local PN clock is delayed by half a chip, and the acquisition process is repeated. After acquisition has been made, SLCU initiates tracking.

\subsubsection{Acquisition}

The block diagram used to implement acquisition (serial search) is as shown in Figure 6. It consists of a square law envelope detector, and an integrate and dump to detect the correlated signal energy at constant test time intervals (dwell time). The output of the integrator is then compared with a threshold voltage and if the threshold is exceeded, the phase of the local PN is corrected and tracking will be initiated, else a phase update signal is sent by the SLCU to try and correct the phase offset.

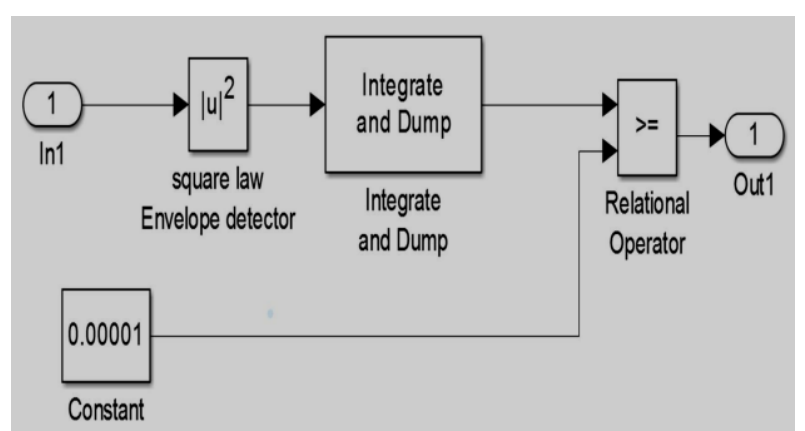

Figure 6. Acquisition subsystem

\subsubsection{Tracking}

The tracking method implemented is the delay locked loop (DLL) method due to ease of implementation and also its quite accurate. The tracking loop consists of two branches as shown in Figure 7.

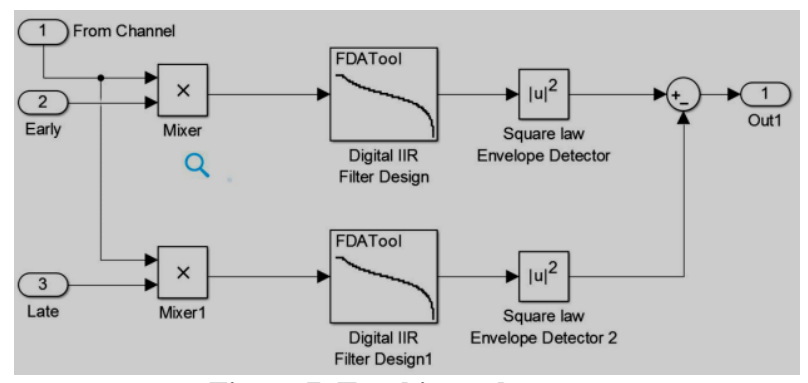

Figure 7. Tracking subsystem

The common input to the lower and upper branch is the input from the AWGN channel. The second input to the upper mixer is the output of the last flip flop of the local PN generator (Early) while the second input to the lower mixer is the output of the second flip flop of the Local PN generator (Late).

Both branches are comprised of a mixer, a digital low pass Butterworth filter and a square law envelope detector. These detects the energy of the late and the early signals coming into the branches at constant dwell times.

The filter used had a sampling frequency of $64 \mathrm{KHz}$ (Nyquist rate) and a cutoff frequency same as the data rate i.e. $1 \mathrm{KHz}$.

The summer at the output of the tracking loop subtracts the two signals to generate and error signal.

\subsubsection{Voltage Controlled Oscillator}

The error signal from the tracking loop drives the digital VCO so as it can correct the clock frequency of $32 \mathrm{KHz}$. The VCO runs at the frequency of the PN generator of the transmitter. The limiter is used to convert the sinusoid from the VCO to a square wave signal. 


\subsubsection{Search and Lock Control Unit}

The block diagram of the SLCU implementation is as shown in Figure 8. The clock produces a pulse at half the chip period i.e. $64 \mathrm{KHz}$ which is inverted and fed into the NAND gate in order to check the status of acquisition system. The NAND outputs either a HIT (acquisition has occurred) so as to proceed with tracking or not. If there is no HIT, a phase replica is tested again until a HIT is gotten.

The T Flip-flop is used to generated a half chip clock at each half chip period in case there is no HIT i.e. speed up the clock. The clock is gotten after modulo two additions of the VCO clock with the T-flip-flop output.

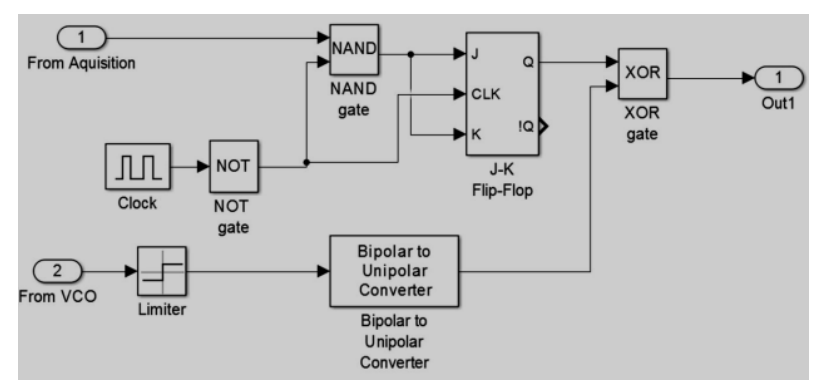

Figure 8. SLCU subsystem

The final clock update will either be at the chip rate (normal speed) if acquisition occurred or at half chip rate (local PN code faster than the received PN code) if acquisition process does not reach it is final decision.

\subsubsection{Local PN Code Generator}

The local PN generator is designed similar to the PN generator in the transmitter except that the clock that runs it came from the output of the SLCU. The early and late sequences are tapped as shown in Figure 9.

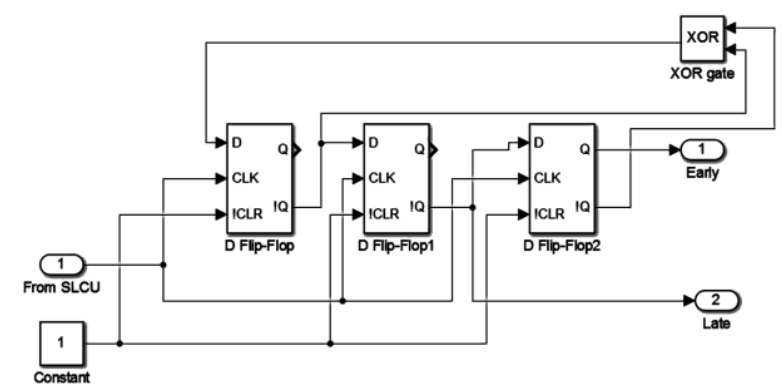

Figure 9. Local PN generator

\subsubsection{BPSK Demodulator}

The baseband BPSK demodulator from the communications blockset with a log-likelihood-ratio decision mode is used. After demodulation, the signal is passed through an integrate and dump to stabilize it, then it is passed through a limiter to get a waveform that is bipolar with 2-ary (from -1 to 1 ) and then the signal is converted to unipolar to get the output of the whole system.

\section{RESULTS}

The results focus on showing the spreading achieved and also despreading. Figure 10. shows user data before spreading and after spreading. we also show the tracking for different values of user frequency as illustrated in the following subsections.
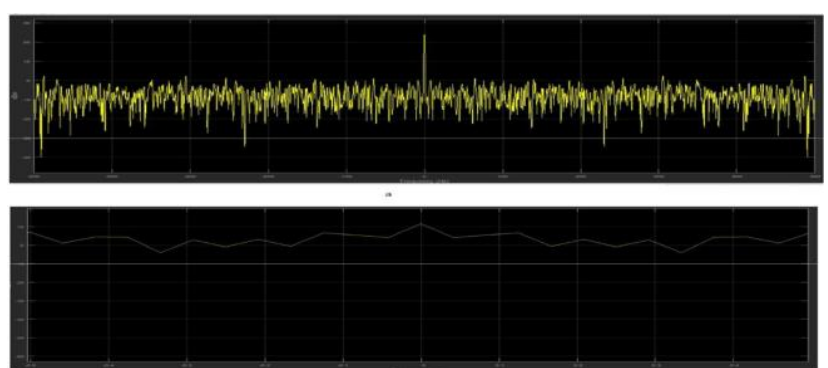

Figure 10. (a) user data before spreading; (b) user data after spreading

\subsection{Scenario 1: User data at $1 \mathrm{KHz}$}

The result for the system with user data at $1 \mathrm{KHz}$ and the spreading signal maintained at $32 \mathrm{KHz}$ is shown in Figure 11. It is seen that the received signal has errors during the early stages of tracking between $0-0.01$ seconds but after that the signals are the same.

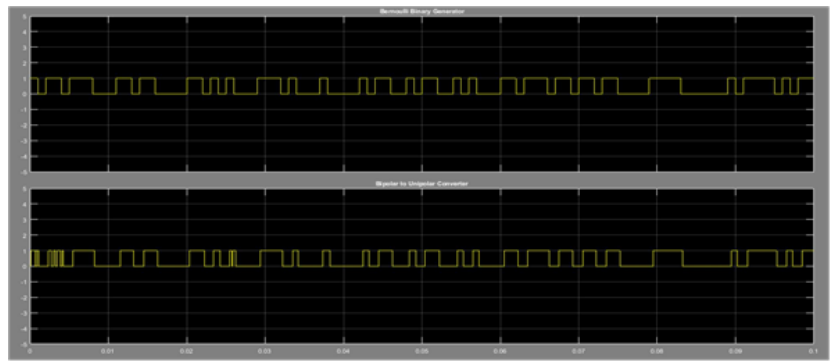

Figure 11. User data at $1 \mathrm{KHz}$

\subsection{Scenario 2: User data at $2 \mathrm{KHz}$}

The result when the user frequency is adjusted to $2 \mathrm{KHz}$ is shown in Figure 12. The system tracks the signal even if the user frequency changes, this only happens if the VCO quiescent frequency is adjusted to match the user frequency.

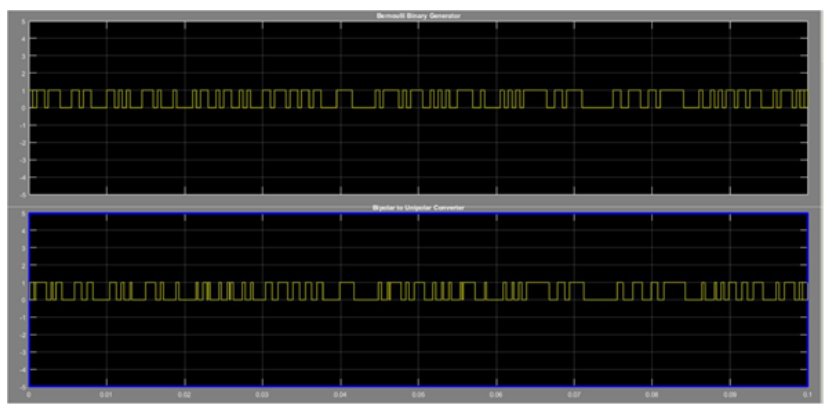

Figure 12. User data at $2 \mathrm{KHz}$ 


\subsection{BER versus SNR}

The performance of the system is investigated by drawing bit error rate (BER) versus signal to noise ratios (SNR) with a digital filter as the correlator and with an integrator as the correlator. The graphs obtained are shown in Figure 13 using a digital filter correlator and Figure 14 using an integrator. From the graphs, it is evident that the integrator performs better than the digital filter. Also, as the SNR is raised, the number of errors recorded reduced for the same amount of sample time.

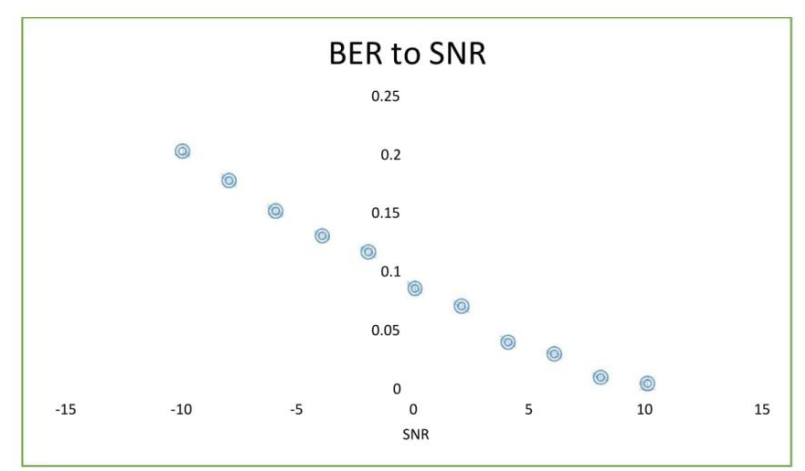

Figure 13. Graph of BER against SNR for a filter as correlator

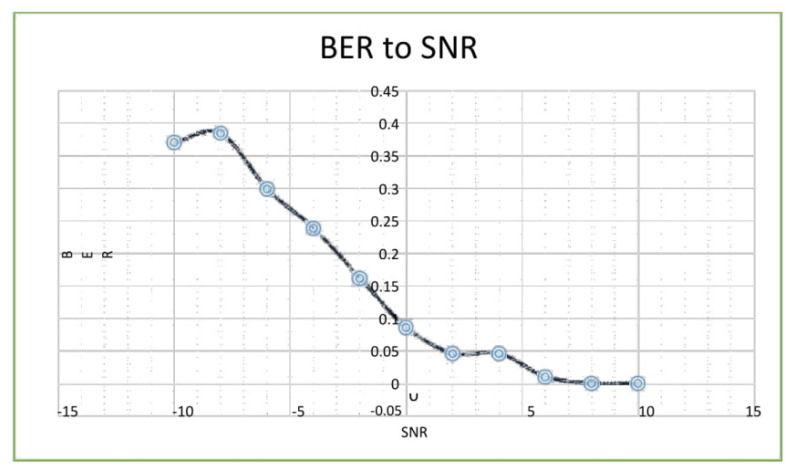

Figure 14. Graph of BER against SNR for an integrator as correlator

\section{CONCLUSION}

The objectives of the paper were achieved. DSSS systems were studied and the importance of code tracking was demonstrated. The importance of an acquisition system was also demonstrated. The system allows the receiver to adjust its clock frequency to match up the locally generated PN sequence with the received PN sequence. The tracking system prevented errors by ensuring the two PN sequences don't go out of synchronization with each other which would result in a loss of signal. The tracking system allowed for demodulation process to be more reliable by allowing a larger amount of signal energy to be received by the demodulator.

\section{REFERENCES}

[1] B. Sklar, Digital communications: fundamentals and applications, Second Edition, Upper Saddle River, New Jersey: Prentice Hall PTR, 2009.

[2] W. Stallings, Data and computer communications, Eighth Edition, Upper Saddle River, New Jersey: Pearson Education Inc., 2007.

[3] M. Simon, J. Omura, R. Scholtz and B. Levitt, Spread spectrum communications handbook, Electronic Edition, McGraw-Hill Inc., 2004.

[4] D. Torrieri, Principles of spread spectrum communication systems, Springer Science Business Media Inc., 2005.

[5] A. Viterbi, CDMA: principles of spread spectrum communications, Addison-Wesley Publishing Company, 1995.

[6] H. Zepernick and A. Finger, Pseudo random signal processing, theory and application, John Wiley \& Sons, 2005.

[7] H. Omar, Tracking loop of direct sequence spread spectrum signals, B.Sc. Graduation Project, University of Nairobi, Kenya, 2016.

[8] B. Agee, R. Kleinman, J. Reed, Soft synchronization of direct sequence spread-spectrum signals, IEEE Transactions on Communications, Vol.44, Issue 11, PP 1527-1536, 1996.

[9] S. Curry, D. Schwartz, J. Collins, State transition assisted DSSS code synchronization, IEEE Military Communications Conference, USA, 1999.

[10] B. Grundlehner, J. Haartsen, S. Rajan, Improved synchronization performance in DSSS systems using concatenated sequence, IEEE $7^{\text {th }}$ Symp. on SpreadSpectrum Tech. and Appl., Czech Republic, 2002.

[11] A. Tayebi, B. Stevan, S. Akshya, Performance analysis of chaotic DSSS-CDMA synchronization under jamming attack, Circuits, Systems, and Signal Processing Vol.35, No12, PP.4350-4371, 2016. 